\title{
Trabecular Metal : vers de nouvelles possibilités en implantologie?
}

\section{Jérôme Surmenian}

\author{
UFR d'Odontologie, Département de Parodontologie, Nice, France \\ surmenianjerome@yahoo.fr
}

La technologie Trabecular Metal est utilisée avec succès depuis une quinzaine d'années en chirurgie orthopédique que ce soit pour des prothèses de hanches, de genoux ou d'épaules.

L'intérêt majeur du Trabecular Metal réside dans sa structure poreuse qui permet une apposition osseuse au sein même de l'implant, introduisant le concept d' "ostéo-pénétration ».

Cette technologie appliquée aux implants dentaires, devrait étendre les possibilités de traitement en cas de faible densité osseuse ou de faible potentiel de cicatrisation.

La présentation commencera par une illustration du procédé d'obtention du Trabecular Metal et de ses indications en chirurgie orthopédique.

Puis nous nous concentrerons sur son utilisation en implantologie dentaire. 